\title{
Self-interacting scalar fields on spacetime with compact hyperbolic spatial part
}

\author{
Andrei Bytsenko, Klaus Kirsten \\ Dipartimento di Fisica, Università degli Studi di Trento \\ 38050 Povo (Trento), Italia \\ Sergei Odintsov ${ }^{\dagger}$ \\ Department of Physics, Faculty of Science, Hiroshima University \\ Higashi-Hiroshima 724, Japan
}

March 1993

Subject classification number: 1100

0460

0230

\begin{abstract}
We calculate the one-loop effective potential of a self-interacting scalar field on the spacetime of the form $\mathbb{R}^{2} \times H^{2} / \Gamma$. The Selberg trace formula associated with a co-compact discrete group $\Gamma$ in $P S L(2, \mathbb{R})$ (hyperbolic and elliptic elements only) is used. The closed form for the one-loop unrenormalized and renormalized effective potentials is given. The influence of non-trivial topology on curvature induced phase transitions is also discussed.
\end{abstract}

\section{Introduction}

The effective action in quantum field theory in curved spacetime is the central object which, in principle, should define the dynamics of the early universe at scales bigger than the Planck scale. In particular, it should be relevant for the description of the inflationary universe (for a review see [1-4]) which may be based on some phase transition (for example induced by temperature [1-4]). Unfortunately there are a lot of difficulties in calculating the effective action in a quantum field theory in a general curved spacetime (for a review see [5). Therefore it is very natural to deal with some specific spaces which are interesting from the cosmological viewpoint. In this context it is significant to investigate the constant curvature compact spaces, contained in the well known class of the so-called Robertson-Walker space-time forms.

The evaluation of the effective potential in self-interacting scalar field theories on curved backgrounds has a long history and many explicit calculations of effective potentials in different spacetimes have been done [6-20] (for a review see [5]). The influence of nontrivial topology on the effective potential has been particularly studied in [17, 18, 19, 6, 14, 15], concentrating however on spacetimes with vanishing curvature. Our main aim is, to analyze the influence of nonvanishing constant curvature together with nontrivial topology (see also [13, 21]). As an example we choose the spacetime $\mathbb{R}^{2} \times H^{2} / \Gamma$, where $\Gamma$ is a co-compact discrete group in $P S L(2, \mathbb{R})$

\footnotetext{
*Permanent address: Department of Theoretical Physics, State Technical University, St. Petersburg 195251, Russia

${ }^{\dagger}$ On leave from Tomsk Pedagogical Institute, 634041 Tomsk, Russia
} 
with hyperbolic and elliptic elements. For this spacetime the Selberg trace formula associated with $\Gamma$ allows us to analyze several properties of the one-loop effective potential. (Note that recently the classification of topologies of hyperbolic universes has been discussed in ref. [22].) The organisation of the paper is as follows. In section 2 the non-renormalized one-loop effective potential for a quartic self-interacting scalar field coupled to gravity is calculated using the Selberg trace formula. In section 3 the renormalization of the one-loop effective potential is performed. The analysis of the phase transition in the model is given in section 4 , including some remarks on scalar electrodynamics on $\mathbb{R}^{2} \times H^{2} / \Gamma$. Finally a short summary of our results is given in section 5 .

\section{Regularized one-loop effective potential}

The concept of the effective action and the effective potential is well discussed in the literature [23-26], so the introduction of these quantities will be very brief. We consider a self-interacting scalar field coupled to gravity on the spacetime of the form $\mathcal{M}=\mathbb{R}^{2} \times H^{2} / \Gamma$. Here the group $\Gamma$ in $P S L(2, \mathbb{R})=S L(2, \mathbb{R}) /\{-1,1\}$ is a discrete co-compact group acting on the two-dimensional Lobachevsky space $H^{2}$, i.e. the signature of $\Gamma$ contains only hyperbolic and elliptic numbers. The bare Lagrangian density of the theory reads

$$
L(x)=-\frac{1}{2}\left(\nabla_{\mu} \phi\right)\left(\nabla^{\mu} \phi\right)-\frac{1}{2}\left(m^{2}+\xi R\right) \phi^{2}-\frac{\lambda}{4 !} \phi^{4}
$$

where $m$ is the mass of the field, $\xi$ is the conformal coupling constant of the quantum scalar field $\phi$ to the gravitational field, represented by the scalar curvature $R$. Because $H^{2} / \Gamma$ is a constant curvature space, the concept of the effective potential is well defined [12]. Formally it is given in the form

$$
V\left(\phi_{c}\right)=V^{(0)}\left(\phi_{c}\right)+\hbar V^{(1)}\left(\phi_{c}\right)+\mathcal{O}\left(\hbar^{2}\right)
$$

with the purely classical part

$$
V^{(0)}\left(\phi_{c}\right)=\frac{1}{2} m^{2} \phi_{c}^{2}+\frac{1}{2} \xi R \phi_{c}^{2}+\frac{\lambda}{4 !} \phi_{c}^{4}
$$

and with the one-loop quantum corrections of the form

$$
V^{(1)}\left(\phi_{c}\right)=\frac{1}{2 \operatorname{vol}(\mathcal{M})} \ln \operatorname{det}\left(\mu^{2} A\right)
$$

where $\mu$ is a normalization constant with dimension of length and the relevant Laplace-like operator $A$ is given by

$$
A=-\Delta+m^{2}+\xi R+\frac{\lambda}{2} \phi_{c}^{2}
$$

Using the zeta function prescription for the regularization of functional determinants [27], [28], equation (2.4) is given in the form (from now on we will write $\phi$ instead of $\phi_{c}$ )

$$
V^{(1)}(\phi)=-\frac{1}{2 \operatorname{vol}(\mathcal{M})}\left[\zeta^{\prime}{ }_{A}(0)+\zeta_{A}(0) \ln \mu^{2}\right]
$$

where $\zeta_{A}(s)$ is the zeta function associated with the operator $A$. This means

$$
\zeta_{A}(s)=\sum_{\vec{k}} \Omega_{\vec{k}}^{-s}
$$


with the eigenvalues $\Omega_{\vec{k}}, \vec{k}=(j, n, l)$, of the operator $A$ given by

$$
\begin{aligned}
\Omega_{\vec{k}} & =\left(\frac{2 \pi}{L} n\right)^{2}+\left(\frac{2 \pi}{\beta} l\right)^{2}+\lambda_{j} R_{H}^{-2}+m^{2}+\xi R+\frac{\lambda}{2} \phi^{2} \\
& =:\left(\frac{2 \pi}{L} n\right)^{2}+\left(\frac{2 \pi}{\beta} l\right)^{2}+\left(r_{j}^{2}+\delta^{2}\right) R_{H}^{-2}, \quad j \in \mathbb{N}_{0}, n, l \in \mathbb{Z}
\end{aligned}
$$

where we introduced the notations $\delta^{2}=\left(M^{2}+\xi R\right) R_{H}^{2}+\frac{1}{4}$ and $M^{2}=m^{2}+\frac{\lambda}{2} \phi^{2}$. Here $\lambda_{j}$ are the eigenvalues of the Laplacian $-\Delta_{H}$ of the hyperbolic spatial part $H^{2} / \Gamma$ of the manifold $\mathcal{M}$, normalized to $R_{H}=1$, where $R=-2 R_{H}^{-2}$.

The contribution of this part of the spectrum of the one-loop effective potential may be determined using the Selberg trace formula. This will be done in the following.

First taking the limit $L, \beta \rightarrow \infty$, we find

$$
\lim _{L, \beta \rightarrow \infty} \frac{\zeta_{A}(s)}{L \beta}=\frac{1}{4 \pi} R_{H}^{2(s-1)} F(s ; \delta)
$$

where we introduced the function

$$
F(s ; \delta)=\frac{1}{s-1} \sum_{j}\left[r_{j}^{2}+\delta^{2}\right]^{1-s}
$$

The series is absolutely convergent for $\operatorname{Re} s>2$. The necessary analytical continuation of equation 2.10) to $s=0$ will be constructed using the Selbergs trace formula. Assuming that $h(r)$ is analytic in the strip $|\operatorname{Im} r| \leq 1 / 2+\epsilon, \epsilon>0$ and that $h(r)=\mathcal{O}\left(\left(1+|r|^{2}\right)^{-1-\epsilon}\right)$ in the strip for $r \rightarrow \infty$, it reads [29], 30], [31]

$$
\begin{aligned}
\sum_{j} h\left(r_{j}\right)= & \frac{V(\mathcal{F})}{4 \pi} \int_{-\infty}^{\infty} d r r \tanh (\pi r) h(r) \\
& +\sum_{\{P\}_{\Gamma}} \sum_{k=1}^{\infty} \frac{\chi^{k}(P) l(P)}{2 \sinh (k l(P))} \hat{h}(k l(P)) \\
& +\sum_{\{Q\}_{\Gamma}} \sum_{k=1}^{n-1} \frac{\chi^{k}(Q)}{2 n \sin \left(\frac{k \pi}{n}\right)} \int_{-\infty}^{\infty} d r \frac{e^{-\frac{2 \pi r k}{n}}}{1+e^{-2 \pi r}} h(r)
\end{aligned}
$$

Here $\mathcal{F}$ is a fundamental domain and its measure can be computed by the Gauss-Bonnet formula [30, $V(\mathcal{F})$ is the volume of the fundamental domain and $\hat{h}$ is the Fourier transform of $h$. The summation $\{P\}_{\Gamma}\left(\{Q\}_{\Gamma}\right)$ is taken over all primitive hyperbolic (elliptic) conjugacy classes in $\Gamma$, $l(P)$ is the length of a closed geodesic associated with the element $P$ of the conjugacy class, $\chi$ is an arbitrary finite-dimensional representation of $\Gamma$ (character of $\Gamma$ ) and finally $n=n(Q)$ is the order of the class with representative $Q$. On the left-hand side the summation is over all solutions $r_{j}$ of the equations $r_{j}^{2}=\lambda_{j}-1 / 4$.

Using equation (2.11), the analytic continuation of equation 2.10) is found to be

$$
\begin{aligned}
F(s ; \delta)= & \frac{V(\mathcal{F})}{4 \pi(s-1)(s-2)} \delta^{2(2-s)}-\frac{V(\mathcal{F})}{2 \pi(s-1)} \int_{0}^{\infty} d r r(1-\tanh \pi r)\left(r^{2}+\delta^{2}\right)^{1-s} \\
& +\frac{(2 \delta)^{\frac{3}{2}-s}}{\sqrt{\pi} \Gamma(s)} \sum_{\{P\}_{\Gamma}} \sum_{k=1}^{\infty} \frac{\chi^{k}(P) l(P)}{2 \sinh (k l(P))}(k l(P))^{s-\frac{3}{2}} K_{s-\frac{3}{2}}(\delta k l(P)) \\
& +\sum_{\{Q\}_{\Gamma}} \sum_{k=1}^{n-1} \frac{\chi^{k}(Q)}{2 n \sin \left(\frac{k \pi}{n}\right)} \int_{-\infty}^{\infty} d r \frac{e^{-\frac{2 \pi r k}{n}}}{1+e^{-2 \pi r}}\left(r^{2}+\delta^{2}\right)^{1-s}
\end{aligned}
$$


The contribution to the effective potential associated with the summation over hyperbolic classes, which we shall denote as $V_{P}$, can be rewritten in terms of the logarithmic derivative of the Selberg type zeta-function

$$
\frac{Z^{\prime}}{Z}(s)=\sum_{\{P\}_{\Gamma}} \sum_{k=1}^{\infty} \frac{\chi^{k}(P) l(P)}{2 \sinh (k l(P))} e^{-\left(s-\frac{1}{2}\right) k l(P)},
$$

in the form [32, 33]

$$
V_{P}=\frac{1}{\Gamma(s-1) \Gamma(2-s)} \int_{0}^{\infty} d y\left(y^{2}+2 y \delta\right)^{1-s} \frac{Z^{\prime}}{Z}\left(y+\delta+\frac{1}{2}\right)
$$

Now using equation (2.9) and equation (2.6) all the information needed for the calculation of the one-loop quantum correction is known, the only thing to do is to find the derivative of equation (2.12) at $s=0$. We will skip this intermediate step and go directly to the effective potential. In order to give the final result, it is useful to introduce some abbreviations. We will use

$$
\begin{aligned}
X & =\int_{0}^{\infty} d r r\left(r^{2}+\delta^{2}\right) \ln \left(1+\frac{r^{2}}{\delta^{2}}\right)(1-\tanh \pi r), \\
H & =\int_{0}^{\infty} d y\left(y^{2}+2 y \delta\right) \frac{Z^{\prime}}{Z}\left(y+\delta+\frac{1}{2}\right), \\
E & =\int_{-\infty}^{\infty} d r \frac{e^{-\frac{2 \pi r k}{n}}}{1+e^{-2 \pi r}}\left(r^{2}+\delta^{2}\right) \ln \left(1+\frac{r^{2}}{\delta^{2}}\right) \\
f(k) & =\frac{\chi^{k}(Q)}{2 n \sin ^{2}\left(\frac{k \pi}{n}\right)} \\
h(k) & =-\frac{1}{4}+\frac{1}{2 \sin ^{2}\left(\frac{k \pi}{n}\right)} \\
g(k) & =\frac{\chi^{k}(Q)}{n \sin \left(\frac{k \pi}{n}\right)}
\end{aligned}
$$

In terms of these, the non-renormalized one-loop effective potential, now including the for our calcutation necessary counterterms [34], [35], 24], [7], is

$$
\begin{aligned}
V(\phi)= & \Lambda+\delta \Lambda+(\kappa+\delta \kappa) R+\frac{1}{2}(\xi+\delta \xi) R \phi^{2}+\frac{1}{2}(a+\delta a) R^{2}+\frac{1}{4 !}(\lambda+\delta \lambda) \phi^{4} \\
& +\frac{1}{2}\left(m^{2}+\delta m^{2}\right) \phi^{2}+V_{\text {hyp }}(\phi)+V_{\text {ell }}(\phi)
\end{aligned}
$$

where for clarity we introduce the hyperbolic (respectively elliptic) contribution $V_{\text {hyp }}(\phi)$ (respectively $\left.V_{\text {ell }}(\phi)\right)$,

$$
\begin{aligned}
V_{\text {hyp }}(\phi)=-\frac{\hbar}{32 \pi^{2} R_{H}^{4}}\left\{\frac{1}{2} \delta^{4}\left[\frac{3}{2}+\ln \left(\frac{\mu^{2} R_{H}^{2}}{\delta^{2}}\right)\right]-2 X+\frac{4 \pi H}{V(\mathcal{F})}\right. \\
\left.+2\left[\frac{1}{24} \delta^{2}+\frac{7}{960}\right]\left[1+\ln \left(\frac{\mu^{2} R_{H}^{2}}{\delta^{2}}\right)\right]\right\} \\
V_{\text {ell }}(\phi)=-\frac{\hbar}{8 \pi R_{H}^{4} V(\mathcal{F})} \sum_{\{Q\}_{\Gamma}} \sum_{k=1}^{n-1} \times \\
\quad\left\{-f(k)\left[\delta^{2}+h(k)\right]\left[1-\ln \left(\frac{\mu^{2} R_{H}^{2}}{\delta^{2}}\right)\right]+g(k) E\right\}
\end{aligned}
$$

In order to remove the dependence on the arbitrary parameter $\mu$, let us now continue with the renormalization procedure. 


\section{Renormalization}

The renormalization of the one-loop effective potential of a self-interacting scalar field in curved spacetime is by now well known (see for example [7], [24], [5]), so our prescription will be brief. We fix the counterterms by the renormalization condition

$$
\begin{aligned}
\xi & =\left.\frac{\partial^{3} V}{\partial R \partial \phi^{2}}\right|_{R=0, \phi=M} \\
a & =\left.\frac{\partial^{2} V}{\partial R^{2}}\right|_{R=0, \phi=M} \\
\lambda & =\left.\frac{\partial^{4} V}{\partial \phi^{4}}\right|_{R=0, \phi=M}
\end{aligned}
$$

For simplicity we restrict to the massless case $m=0$ (so no $\Lambda$ and $\kappa$ renormalization is necessary), because our main aim is to analyze the influence of the curvature and toplogy on the phase transition. But in principle, nothing can prevent us from adding a nonvanishing mass, the calculation would only be slightly more difficult.

Using equation (2.15), the counterterms are found to be

$$
\begin{aligned}
& \delta a=\frac{\hbar}{128 \pi^{2}}\left\{-4\left[\left(\xi-\frac{1}{6}\right)^{2}+\frac{1}{180}\right] \ln \left(\frac{\lambda M^{2}}{2 \mu^{2}}\right)\right.\left.+\frac{8 \pi}{V(\mathcal{F})} \sum_{\{Q\}_{\Gamma}} \sum_{k=1}^{n-1} f(k)[\epsilon+h(k)] \ln \left(\frac{\lambda M^{2}}{2 \mu^{2}}\right)\right\} \\
& \delta \lambda=-\frac{\hbar \lambda^{2}}{32 \pi^{2}}\left[8+3 \ln \left(\frac{\lambda M^{2}}{2 \mu^{2}}\right)\right] \\
& \delta \xi=-\frac{\hbar \lambda}{32 \pi^{2}}\left[2+\ln \left(\frac{\lambda M^{2}}{2 \mu^{2}}\right)\right]\left\{\xi-\frac{1}{6}+\frac{2 \pi}{V(\mathcal{F})} \sum_{\{Q\}_{\Gamma}} \sum_{k=1}^{n-1} f(k)\right\}
\end{aligned}
$$

It is seen, that the given counterterms differ from the counterterms derived in [7] for a selfinteracting scalar field theory on a smooth manifold. This may be traced back to the co-compact group $\Gamma$ under consideration, due to which the manifold $H^{2} / \Gamma$ is no longer a smooth one.

Introducing $\epsilon=\xi-\frac{1}{8}$, after some calculation the final result for the one-loop renormalized effective potential reads

$$
\begin{aligned}
V_{r}(\phi)= & \frac{1}{2} \xi R \phi^{2}+\frac{1}{2} a R^{2}+\frac{\lambda}{4 !} \phi^{4} \\
& -\frac{\hbar}{128 \pi^{2}} \times \\
& \left\{\frac{25}{12} \lambda^{2} \phi^{4}-\lambda \phi^{2} R\left[\frac{9}{8}-7 \xi\right]+R^{2}\left[3 \xi^{2}-\frac{11}{12} \xi+\frac{79}{960}\right]\right. \\
& -2 X R^{2}+\frac{4 \pi H R^{2}}{V(\mathcal{F})} \\
& -\left[\frac{1}{2} \lambda^{2} \phi^{4}+2 \lambda \phi^{2} R\left(\xi-\frac{1}{6}\right)+2 R^{2}\left(\left(\xi-\frac{1}{6}\right)^{2}+\frac{1}{180}\right)\right] \times \\
& +\frac{4 \pi}{V(\mathcal{F})} \sum_{\{Q\}_{\Gamma}} \sum_{k=1}^{n-1} \times
\end{aligned}
$$




$$
\begin{aligned}
& {\left[3 \lambda \phi^{2} R f(k)-R^{2} f(k)(\epsilon+h(k))+R^{2} g(k) E\right.} \\
& \left.\left.-f(k)\left(\lambda \phi^{2} R-[\epsilon+h(k)] R^{2}\right)\left(\ln \left(\frac{\phi^{2}}{M^{2}}\right)+\ln \left(1-\frac{\epsilon R}{\lambda \phi^{2}}\right)\right)\right]\right\}
\end{aligned}
$$

For the subsequent analysis of the phase transition, let us consider several limits. The small curvature case is very easily extracted from equation (3.3), we find

$$
\begin{aligned}
V_{r}(\phi)= & \frac{1}{2} \xi R \phi^{2}+\frac{1}{2} a R^{2}+\frac{\lambda}{4 !} \phi^{4} \\
& -\frac{\hbar}{128 \pi^{2}} \times \\
& \left\{\frac{1}{2} \lambda^{2} \phi^{4}\left[\frac{25}{6}-\ln \left(\frac{\phi^{2}}{M^{2}}\right)\right]\right. \\
& +R \lambda \phi^{2}\left[3-\ln \left(\frac{\phi}{M^{2}}\right)\right] \times \\
& -R^{2} \ln \left(\frac{\phi^{2}}{M^{2}}\right) \times \\
& {\left[\left(\xi-\frac{1}{6}\right)+\frac{4 \pi}{V(\mathcal{F})} \sum_{\{Q\}_{\Gamma}} \sum_{k=1}^{n-1} f(k)\right] } \\
& {\left.\left[\left(\xi-\frac{1}{6}\right)^{2}+\frac{1}{180}\right)-\frac{4 \pi}{V(\mathcal{F})} \sum_{\{Q\}_{\Gamma}} \sum_{k=1}^{n-1} f(k)(\epsilon+h(k))\right] } \\
& \left.+\mathcal{O}\left(R^{3}\right)\right\}
\end{aligned}
$$

Let us once more stress the importance of the presence of the elliptic elements of $\Gamma$ leading to obvious contributions in (3.4). The small background field limit is more difficult to obtain. We will not state explicitly the constant part $\Lambda_{\text {eff }}$ of the potential (the so called cosmological constant), but concentrate only on the quadratic contribution. Introducing the functions

$$
\begin{aligned}
& C(n)=\int_{0}^{\infty} d r r^{n}(1-\tanh \pi r) \ln \left(\epsilon+r^{2}\right) \\
& F(n)=\int_{-\infty}^{\infty} d r r^{n} \frac{e^{-\frac{2 \pi r k}{n}}}{1+e^{-2 \pi r}} \ln \left(\epsilon+r^{2}\right) \\
& G(n)=\int_{0}^{\infty} d y\left(y^{2}+2 y \sqrt{\epsilon}\right)^{n} \frac{Z^{\prime}}{Z}\left(y+\sqrt{\epsilon}+\frac{1}{2}\right)
\end{aligned}
$$

the final expansion may be given in the form

$$
\begin{aligned}
V(\phi)= & \Lambda_{\text {eff }}+\frac{1}{2} \xi R \phi^{2} \\
& -\frac{\hbar \lambda R}{128 \pi^{2}} \phi^{2} \times \\
& \left\{7 \xi-\frac{9}{8}-\frac{1}{12} \ln \epsilon+2 C(1)+\frac{4 \pi G(0)}{V(\mathcal{F})}\right. \\
& +2\left(\xi-\frac{1}{6}\right) \ln \left(-\frac{\lambda M^{2}}{\epsilon R}\right)+\frac{2}{\epsilon}\left(\left(\xi-\frac{1}{6}\right)^{2}-\frac{1}{576}\right) \\
& \left.+\frac{4 \pi}{V(\mathcal{F})} \sum_{\{Q\}_{\Gamma}} \sum_{k=1}^{n-1}\left[f(k)\left(2+\ln \left(-\frac{\lambda M^{2}}{R}\right)\right)-g(k) F(0)\right]\right\} \\
& +\mathcal{O}\left(\phi^{4}\right)
\end{aligned}
$$


Armed with these results let us now continue with the analysis of the phase transition in the considered theory.

\section{Curvature induced phase transition}

In this section we will try to analyze the curvature induced phase transitions in our model, taking into account the topology effects. Let us first work in linear curvature approximation with the effective potential (3.4),

$$
\begin{aligned}
V_{r}(\phi)= & \frac{\lambda}{4 !} \phi^{4}-\frac{1}{2} \xi|R| \phi^{2}+\frac{\lambda^{2} \phi^{4}}{256 \pi^{2}}\left[\ln \left(\frac{\phi^{2}}{M^{2}}\right)-\frac{25}{6}\right] \\
& -\frac{\lambda|R| \phi^{2}}{64 \pi^{2}}\left[\ln \left(\frac{\phi^{2}}{M^{2}}\right)-3\right]\left[\left(\xi-\frac{1}{6}\right)+T\right]
\end{aligned}
$$

where $T=(2 \pi / V(\mathcal{F})) \sum_{\{Q\}_{\Gamma}} \sum_{k=1}^{n-1} f(k)$. We took into account that the curvature $R$ is negative, $R=-2 / R_{H}^{2}$, hence $|R|=2 / R_{H}^{2}$.

Let us discuss now the possibility of curvature induced phase transition of first order. In this case the order parameter $\langle\phi\rangle$ at some critical curvature $R_{c}$ is quickly changed. The general theory of such phase transitions in linear curvature approximation has been developed in [36], [5]. Following these references, we introduce the dimensionless variables $x=\phi^{2} / M^{2}, y=|R| / M^{2}$. In these variables, the effective potential (4.1) is given in the form

$$
\begin{aligned}
\frac{V}{M^{4}}= & \frac{\lambda x^{2}}{4 !}-\frac{1}{2} \xi y x+\frac{\lambda^{2} x^{2}}{256 \pi^{2}}\left(\ln x-\frac{25}{6}\right) \\
& -\frac{\lambda x y}{64 \pi^{2}}(\ln x-3)\left[\left(\xi-\frac{1}{6}\right)+T\right]
\end{aligned}
$$

Note that the topological correction $T$ appears on the equal foot with the parameter $\xi$ in the quantum correction to the effective potential.

The standard conditions of the first-order phase transitions are

$$
V\left(x_{c}, y_{c}\right)=0,\left.\quad \frac{\partial V}{\partial x}\right|_{x_{c}, y_{c}}=0,\left.\quad \frac{\partial^{2} V}{\partial x^{2}}\right|_{x_{c}, y_{c}}>0
$$

Now, one can analyze the conditions (4.3) for the potential (4.2) along the analysis given [36]. The result of this analysis is the following. The first two conditions of (4.3) for the effective potential (4.2) are fulfilled. However, the leading term in $\partial^{2} V /\left.\partial x^{2}\right|_{x_{c}, y_{c}}$ is cancelled,

$$
\left.\frac{\partial^{2} V}{\partial x^{2}}\right|_{x_{c}, y_{c}}=0+\mathcal{O}\left(\lambda^{2}\right)
$$

Hence, the one-loop approximation is not enough to answer the question on the possibility of gravitational phase transition in linear curvature approximation. (The non-leading $\mathcal{O}\left(\lambda^{2}\right)$-term in (4.4) which in our one-loop analysis is negative, can be modified by two-loop corrections. Hence, the quite complicated two-loop effective potential is necessary in order to answer the question about the possibility of the first-oder phase transition.)

In order to estimate still the influence of the topology on the phase transitions we will consider now the scalar electrodynamics. Of course, we are not going to repeat the above analysis of the effective potential calculation in that case because it is not so straightforward and quite complicated from the technical viewpoint. Instead we will use the general structure of the effective potential in the one-loop approximation (that we already know from (3.4)). 
Then, the one-loop effective potential is given by (for simplicity we work in Landau gauge),

$$
\begin{aligned}
V_{r}(\varphi)= & \frac{\lambda}{4 !} \varphi^{4}+\frac{1}{(16 \pi)^{2}}\left(\frac{10}{9} \lambda^{2}+12 e^{4}\right)\left(\ln \frac{\varphi^{2}}{M^{2}}-\frac{25}{6}\right) \varphi^{4}-\frac{1}{2} \xi|R| \varphi^{2} \\
& -\frac{|R| \varphi^{2}}{(4 \pi)^{2}}\left[\ln \left(\frac{\varphi^{2}}{M^{2}}\right)-3\right]\left\{\frac{1}{3} \lambda\left[\left(\xi-\frac{1}{6}\right)+T_{1}\right]+\frac{1}{4} e^{2}\left(1+T_{2}\right)\right\}
\end{aligned}
$$

Here the first two terms represent the Coleman-Weinberg potential [25], $e^{2}$ is the electrical charge, $\varphi^{2}=\phi^{\dagger} \phi, T_{1}, T_{2}$ are topological corrections of a similar nature as in (4.2). We don't need their exact form here, but $T_{1}$ should coincide with $T$ in (4.2) up to some overall coefficient. One natural choice which is often used for estimations in flat space [25] is $\lambda \sim e^{4}$. Then (4.5) simplifies, but it still contains the topological correction $T_{2}$. The result of the analysis of equation (4.3) shows that gravitational phase transition is possible with the critical values

$$
x_{c} \approx \exp (-1), \quad y_{c} \approx-\frac{24 e^{4}}{\xi(16 \pi)^{2}} x_{c}, \quad \xi<0
$$

Hence, the topological corrections do not influence the gravitational phase transition in linear curvature approximation.

Now let us discuss again $\lambda \phi^{4}$-theory. The small $\phi$-expansion (3.5) has the typical form

$$
V_{r}(\phi)=\frac{\text { const }}{R_{H}^{4}}+R_{H}^{-2} \phi^{2} \lambda A+\mathcal{O}\left(\phi^{4}\right)
$$

where $A$ is easily obtained from (3.5) and has a quite complicated form. One can see [37], when $A$ changes the sign there is a second-order phase transition in the theory. In this case the critical curvature radius ${ }_{c} R_{H}^{2}$ at which the phase transition occurs depends strongly on the topology. Explicitly we find

$$
{ }_{c} R_{H}^{2}=\frac{2}{\lambda M^{2}} \exp \left\{\frac{32 \pi^{2} B}{\hbar \lambda[\xi-1 / 6+T]}\right\}
$$

with

$$
\begin{aligned}
B= & \xi-\frac{\hbar \lambda}{64 \pi^{2}} \times \\
& \left\{7 \xi-\frac{9}{8}-\frac{1}{12} \ln \varepsilon+2 C(1)+\frac{4 \pi}{V(\mathcal{F})} G(0)\right. \\
- & 2\left(\xi-\frac{1}{6}\right) \ln \varepsilon+\frac{2}{\varepsilon}\left(\left(\xi-\frac{1}{6}\right)^{2}-\frac{1}{576}\right) \\
+ & \left.4 T-\frac{4 \pi}{V(\mathcal{F})} \sum_{\{Q\}_{\Gamma}} \sum_{k=1}^{n-1} f(k) g(k) F(0)\right\}
\end{aligned}
$$

showing the relevance of the topology on the transition point ${ }_{c} R_{H}^{2}$.

\section{Conclusions}

In this paper we considered a self-interacting scalar field coupled to gravity on the spacetime of the form $\mathbb{R}^{2} \times H^{2} / \Gamma$. First we concentrated on the calculation of the one-loop effective potential of the theory given in equation (3.3). Using this potential, it was possible to analyze the first order phase transition in the linear curvature approximation and the second order phase transition in the small background field limit. Whereas for the first order phase transition the 
influence of the topology in the one-loop approximation is not visible (this remark is also true for the scalar electrodynamics on $\left.\mathbb{R}^{2} \times H^{2} / \Gamma\right)$, we find that the critical curvature ${ }_{c} R_{H}^{2}$ for the second order phase transition strongly depends on the topology, (4.8).

Note that as a by-product of our result regarding the renormalization of the effective potential, one can easily get the well-known running coupling constants

$$
\begin{aligned}
\lambda(t) & =\frac{\lambda}{1-\frac{3 \lambda t}{16 \pi^{2}}} \\
\xi(t) & =\frac{1}{6}+\left(\xi-\frac{1}{6}\right)\left(1-\frac{3 \lambda t}{16 \pi^{2}}\right)^{-\frac{1}{3}}
\end{aligned}
$$

The interpretation of the scaling parameter $t$ as a parameter of the scale transformation of the metric $g_{\mu \nu} \rightarrow e^{-2 t} g_{\mu \nu}$ has been given in [38-43] (for a general review see [5]). As one can see, when $t \rightarrow-\infty$ (infrared region) the $\lambda \phi^{4}$ theory is asymptotically free and asymptotically conformally invariant.

\section{Acknowledgements}

We thank Guido Cognola, Luciano Vanzo and Sergio Zerbini for helpful discussions. A.A. Bytsenko and K. Kirsten are grateful to the Faculty of Science and the Department of Physics of the University of Trento for kind hospitality. S.D. Odintsov thanks JSPS (Japan) for financial support. 


\section{References}

[1] Kolb E W and Turner M S. The Early Universe. Addison-Wesley, (1990).

[2] Kolb E W. Phys. Scr. T, 36, 199, (1991).

[3] Linde A D. Particle Physics and Inflationary Cosmology. Harwood Academic, New York, (1990).

[4] Olive K. Phys. Rep., 190, 309, (1990).

[5] Buchbinder I L, Odintsov S D, and Shapiro I L. Effective Action in Quantum Gravity. IOP Publishing, Bristol and Philadelphia, (1992).

[6] Denardo G and Spallucci E. Nuovo Cim. A, 64, 27, (1981).

[7] O'Connor D J and Hu B L. Phys. Rev. D, 30, 743, (1984).

[8] O'Connor D J and Hu B L. Phys. Rev. D, 34, 2535, (1986).

[9] O'Connor D J, Hu B L, and Shen T C. Phys. Lett. B, 130, 31, (1983).

[10] Futamase T. Phys. Rev. D, 29, 2783, (1984).

[11] Huang W-H. Phys. Rev. D, 42, 1287, (1990).

[12] Berkin A L. Phys. Rev. D, 46, 1551, (1992).

[13] Kennedy G. Phys. Rev. D, 23, 2884, (1981).

[14] Actor A. Class. Quantum Grav., 7, 1463, (1990).

[15] Elizalde E and Romeo A. Phys. Lett. B, 244, 387, (1990).

[16] Ford L H and Yoshimura T. Phys. Lett. A, 70, 89, (1979).

[17] Ford L H. Phys. Rev. D, 21, 933, (1980).

[18] Toms D J. Phys. Rev. D, 21, 2805, (1980).

[19] Hosotani Y. Phys. Rev. D, 20, 2783, (1984).

[20] Shore G M. Ann. Phys. (NY), 128, 376, (1980).

[21] Chang P and Dowker J S. Vacuum energy on orbifold factors of spheres. Preprint, Manchester University 1992.

[22] Koike T, Tanimoto U, and Hosoya A. Preprint TIT/HEP-208 December 1992.

[23] Brandenberger R H. Rev. Mod. Phys., 57, 1, (1985).

[24] Buchbinder I L, Odintsov S D, and Shapiro I L. Rivista Nuovo Cim., 12, 1, (1989).

[25] Coleman S and Weinberg E J. Phys. Rev. D, 7, 1888, (1973).

[26] Jackiw R. Phys. Rev. D, 9, 1686, (1974).

[27] Hawking S W. Commun. Math. Phys., 55, 133, (1977).

[28] Critchley R and Dowker J S. Phys. Rev. D, 13, 3224, (1976). 
[29] Selberg A. J. Indian Math. Soc., 20, 47, (1956).

[30] Venkov A B. Proc. Steklov Inst. Math., 4, 1, (1982).

[31] Hejhal D A. The Selberg Trace Formula for PSL(2,R), Vol. I,II. Springer-Verlag, Berlin, (1976).

[32] Bytsenko A A and Zerbini S. Class. Quant. Grav., 9, 1365, (1992).

[33] Bytsenko A A, Vanzo L, and Zerbini S. Mod. Phys. Lett. A, 7, 397, (1992).

[34] Utiyama R and De Witt B. J. Math. Phys., 3, 608, (1962).

[35] Toms D J. Phys. Rev. D, 26, 2713, (1982).

[36] Buchbinder I L and Odintsov S D. Class. Quantum Grav., 2, 721, (1985).

[37] Allen B. Nucl. Phys. B, 226, 228, (1983).

[38] Nelson B L and Panangaden P. Phys. Rev. D, 25, 10, (1982).

[39] Buchbinder I L and Odintsov S D. Izv. VUZov. Fis. (Sov. Phys. J.), 8, 50, (1983).

[40] Buchbinder I L and Odintsov S D. Izv. VUZov. Fis. (Sov. Phys. J.), 12, 108, (1983).

[41] Buchbinder I L and Odintsov S D. Yad. Fiz. (J. Nucl. Phys.), 40, 1338, (1984).

[42] Parker L and Toms D J. Phys. Rev. D, 29, 1584, (1984).

[43] Parker L and Toms D J. Phys. Rev. Lett., 52, 1269, (1984). 